\title{
Green's Function of a Dielectric Slab Grounded by Carbon Fiber Composite Materials
}

\author{
Aidin Mehdipour, Member, IEEE, Abdel-Razik Sebak, Fellow, IEEE, and \\ Christopher W. Trueman, Senior Member, IEEE
}

\begin{abstract}
The exact solution is obtained for the Green's function of an infinitesimal horizontal electric dipole on a dielectric slab backed by a ground plane of carbon fiber composite (CFC) material. We consider both reinforced continuous carbon fiber (RCCF) CFC and carbon nanotube (CNT) CFC. RCCF is modeled by an electrically anisotropic surface impedance tensor whereas CNT is modeled as isotropic. The spectral domain method is used and the numerical integration details particularly dealing with low-converged tail of the integrand for fields at the air-dielectric interface are addressed. Numerical results based on this method compare well with results based on a time-domain finite integration technique. The effect of conductivity and anisotropy of the composite ground plane on electric field is investigated.
\end{abstract}

Index Terms-Anisotropic ground plane, carbon fiber composites (CFC), dielectric slab, Green's function, lossy ground plane.

\section{INTRODUCTION}

A DVANCED carbon-fiber composite (CFC) materials are being used widely in the avionic and automobile industry to replace metals, because of their higher strength, lower weight, and lower cost [1], [2]. The high oxidation stability and high heat-transfer ability allow CFCs to be used for applications where metals would suffer corrosion. However, CFCs have lower electrical conductivity than metals, and so the shielding effectiveness is of concern when electromagnetic compatibility (EMC) must be maintained [3], [4]. There are two types of highly-conductive CFCs, namely the reinforced continuous carbon fiber (RCCF) [3] and the carbon nanotube (CNT) composites [4]-[8]. In one-layer unidirectional RCCF composites, the carbon fibers run in one direction only so that the conductivity is anisotropic, being high along the direction of the fibers, but low in the perpendicular direction. Very recently, we explored the use of RCCF to build radio

Manuscript received $* * * * * *$. This work was supported in part by the Natural Sciences and Engineering Research Council of Canada (NSERC).

A. Mehdipour was with the Department of Electrical and Computer Engineering, Concordia University, Montreal Quebec, H3G 2W1, Canada. He is now with INRS, University of Quebec, Montreal, QC H3C 3J7, Canada (e-mail:mehdipour@emt.inrs.ca).

A. R. Sebak and C. W. Trueman are with the Department of Electrical and Computer Engineering, Concordia University, Montreal Quebec H3G 2W1 Canada. A. R. Sebak is also with PSATRI, King Saud University, Riyadh 11451, KSA. (email: abdo@ece.concordia.ca, trueman@ece.concordia.ca). frequency identification (RFID) antennas [9] and wideband antennas [10]. Unlike RCCF composites, the CNT composite material is an isotropic medium because the CNTs are randomly oriented throughout the material. Being compatible with inkjet printing, CNT is of high interest for antenna fabrication [11], [12]. CNT composites can be made using single-wall nanotubes (SWCNT) [5] or multiwall nanotubes (MWCNT) [6] to obtain conductivity. CNTs are also used in high speed integrated circuits [13] when their high thermal conductivity supports high heat transfer.

There are many types of antennas mounted on or integrated to the surface of vehicles for communication systems, radar, navigation, surveillance and so on. Showing low drag and being suitable for conformal applications, planar microstrip antennas are a very good candidate to be installed on such vehicles [14]. In many cases, the body of vehicle is used as the ground plane for the antenna. There is a very limited work on a grounded slab with lossy or composite material. For example, in [15] the resonance frequency of a rectangular patch antenna on a multilayer composite ground plane is studied using the Galerkin's method. However, the solution is limited to a specific choice of basis functions for better converging integrals which does not apply to general cases when the structure is excited by point sources. Moreover, the complete model of composites is used which lead to a complicated solution and a larger matrix size.

From the EMC point of view, the crosstalk between transmission lines and the coupling between printed wire antennas on the substrate would be another issue which needs to be addressed. Hence, evaluating the Green's function of a dielectric slab with a composite ground plane when both source and observation points located at the air-dielectric interface becomes imperative. Many papers investigate the Green's function of a dielectric slab backed by a perfect electric conductor (PEC) as a ground plane [16]-[20]. In [20], an imperfectly-conducting ground plane is considered, but the small ohmic loss approximation (conductivity $>10^{6} \mathrm{~S} / \mathrm{m}$ ) is used for EM field calculation, showing that the fields at the air-dielectric interface do not depend on the conductivity of ground plane. However, the ohmic loss of CFCs, with a conductivity of about $10^{4} \mathrm{~S} / \mathrm{m}$, is much higher than copper or other kinds of metals. Furthermore, in addition to the high loss, RCCF is also an anisotropic material, making the solution completely different than that for an isotropic ground plane. 


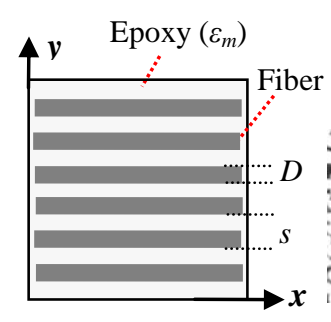

(a)

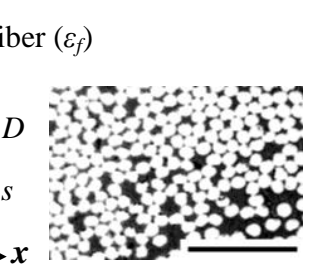

(b)

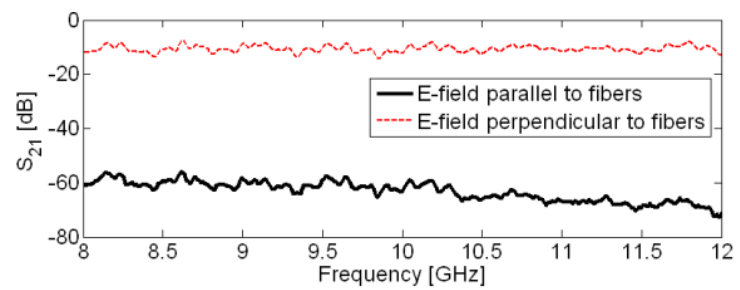

(d)

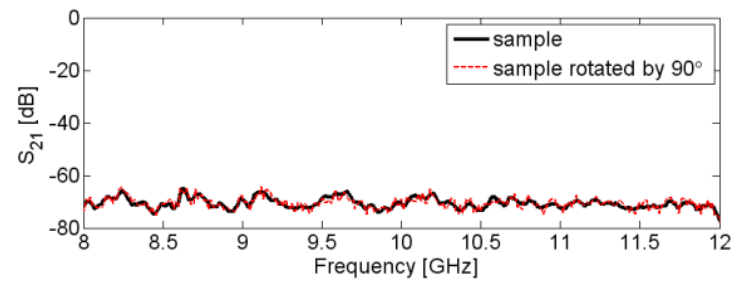

(e)

Fig. 1. (a) RCCF composite, (b) cross-view optical micrograph of RCCF composite (scale bar $50 \mu \mathrm{m}$ ), (c) SEM micrographs of MWCNT composites with 8 wt\% loading [7], (d) measured magnitude of $S_{21}$ parameter for RCCF composite sample, (e) measured magnitude of $S_{21}$ parameter for $8 \%$ MWCNT composite.

In this work, using the spectral domain method, we calculate the exact solution for the Green's function of a dielectric slab backed by a CFC ground plane and excited by a horizontal electric dipole (HED). The assumed CFCs are efficiently modeled by the corresponding surface impedance sheets; a lossy anisotropic sheet for RCCF and a lossy isotropic sheet for CNT. Due to the high conductivity of CFCs, the skin depth would be usually much smaller than the composite thickness. Therefore, the proposed surface impedance model can be used, leading to much simpler and faster solution compared to the case that the complete model of composites is considered. The new expression for the electric field is obtained and the numerical integration is addressed in detail. By using the surface impedance model for the CFC ground plane, the asymptotic part of the integrand becomes similar to that for the PEC ground plane, and the convergence problem is overcome. Finally, some numerical results are presented and compared to that of the slab with a PEC ground plane.

\section{RCCF AND CNT COMPOSITE MATERIALS}

\section{A. RCCF Composite}

Figure 1(a) shows a typical RCCF composite material. The carbon fibers embedded in the epoxy resin are oriented in a specific direction. The effective complex permittivity of onelayer composite depends on the thickness of layer $(t)$, fibers
TABLE I

THICKNESS TO SKIN DEPTH RATIO OF FABRICATED RCCF SAMPLES

\begin{tabular}{|c|c|c|c|}
\hline Composite Material & $\mathbf{5 ~ G H z}$ & $\mathbf{1 0 ~ G H z}$ \\
\hline \multirow{2}{*}{ RCCF } & $x$-axis & 25 & 36.35 \\
\cline { 2 - 4 } & $y$-axis & 2.22 & 3 \\
\hline \multirow{2}{*}{ RCCF + 2\% MWCNT } & $x$-axis & 33.33 & 50.63 \\
\cline { 2 - 4 } & $y$-axis & 6.89 & 9.75 \\
\hline
\end{tabular}

TABLE II

THICKNESS TO SKIN DEPTH RATIO OF FABRICATED CNT SAMPLES

\begin{tabular}{|c|c|c|c|}
\hline Composite Material & $\boldsymbol{\sigma}(\mathbf{S} / \mathbf{m})$ & $\mathbf{5 ~ G H z}$ & $\mathbf{1 0 ~ G H z}$ \\
\hline $\mathbf{4 \%}$ MWCNT & 110 & 5.22 & 7.38 \\
\hline $\mathbf{8 \%}$ MWCNT & 215 & 7.29 & 10.3 \\
\hline SWCNT Buckypaper & 40000 & 98.6 & 139.44 \\
\hline
\end{tabular}

diameter $(D)$, the period of the fibers $(s)$ and complex permittivity of fibers and host medium $\left(\varepsilon_{f}, \varepsilon_{m}\right)$. The effective complex permittivity of homogenized model of RCCF is given by [3]

$$
\begin{gathered}
\varepsilon_{x}=\varepsilon_{z}=(1-g) \varepsilon_{m}+g \varepsilon_{f} \\
\varepsilon_{y}^{-1}=(1-g) \varepsilon_{m}^{-1}+g \varepsilon_{f}^{-1}
\end{gathered}
$$

where $g=\pi D^{2} / 4 s t$ is a coefficient which depends on the volume fraction of fibers inside host medium. The conductivity of material is included in the imaginary part of the complex permittivity. The cross-sectional view of a fabricated RCCF is shown in Fig. 1(b). The average diameter of the fibers is $5 \mu \mathrm{m}$ and separation distance between the fibers is typically $1 \mu \mathrm{m}$. The conductivity of RCCF is high along the direction of fibers $\left(\sigma_{x}\right)$, but low in the perpendicular direction $\left(\sigma_{y}\right)$ so that $\sigma_{x} / \sigma_{y}$ $>1$. We use standard rectangular waveguides to measure the scattering parameters of the RCCF sample with the fibers parallel and perpendicular to the $T E_{10}$ electric field vector. The magnitude of $S_{21}$ parameter of a fabricated sample over Xband frequency range is shown in Fig 1(d). It is observed that RCCF composite blocks the component of the electric field parallel to the fibers much more than the perpendicular component. We have measured the conductivity tensor of some RCCF samples produced by Concordia Center for Composites (CONCOM) [21] over G- and X-band frequency ranges. The values for $\sigma_{x}$ and $\sigma_{y}$ were found to be in the range of 1000-2000 S/m and 10-100 S/m, respectively. Recently, in order to enhance the conductivity of RCCF composite, we added a small volume fraction of MWCNTs to the RCCF material which significantly improves the conductivity in both $x$ and $y$ directions [10]. Table I shows the ratio of sample thickness to skin depth for both fabricated RCCF and RCCF/MWCNT composites. The thickness of samples is about $4 \mathrm{~mm}$.

\section{B. CNT Composites}

High electrical conductivity and high aspect ratio (AR) make the CNTs one of the most promising filler materials for conductive polymer composites. The electrical conductivity of CNT composites depends on the properties and loading of the CNTs, the aspect ratio of the CNTs, and the characteristics of the conductive network throughout the matrix [7]. In order to 


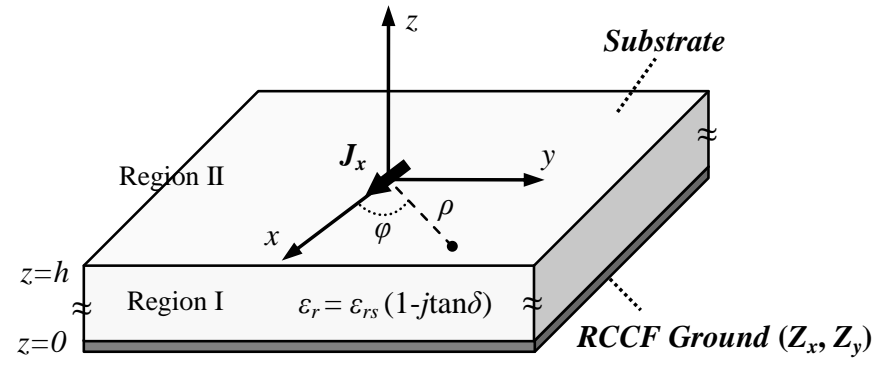

Fig. 2. HED located on the slab grounded by RCCF composite material.

obtain a conductive network, CNTs have to be dispersed efficiently into the resin by shear mixing or by ultrasonic processing. The CNT composites are assumed to have homogeneous isotropic effective complex permittivity [4], [12]. The CNT composite materials are usually non-magnetic. Figure 1(c) shows that CNTs are homogeneously dispersed and oriented in random directions in the host medium, making the composite material electrically isotropic. Figure 1(e) shows the magnitude of $S_{21}$ parameter of a fabricated $8 \%$ MWCNT sample over X-band frequency range for two orientation angles. It is observed that the electric field is blocked almost the same at both $x$ and $y$ directions, showing the isotropy of sample $\left(\sigma_{x} \approx \sigma_{y}\right)$.

The measured conductivity of the sample is $240 \mathrm{~S} / \mathrm{m}$, which is quite high for a loading of just $8 \%$ [7]. The conductivity can be tailored by changing the loading percentage of CNTs so that it can be changed by as much as $40000 \mathrm{~S} / \mathrm{m}$. The ratio of sample thickness to skin depth for some fabricated CNT samples are given in Table II. The thickness of samples is about $3.5 \mathrm{~mm}$. The SWCNTs buckypaper has high electrical conductivity around $70000 \mathrm{~S} / \mathrm{m}$. The buckypaper is a flexible and soft material that needs to be hardened by resin infiltration to be printed on substrate or slabs [12]. During the infiltration the buckypaper expands, which causes a drop in conductivity to a typical $40000 \mathrm{~S} / \mathrm{m}$.

\section{HED ON DIELECTRIC SLAB GROUNDED BY RCCF COMPOSITE}

Figure 2 shows an $x$-directed HED on a dielectric slab of infinite extent in the transverse direction, backed by an RCCF sheet. Because of its high conductivity, the RCCF sheet can be modeled by an anisotropic impedance surface $\left(Z_{x}, Z_{y}\right)$ as follows

$$
Z_{i}=(1+j) \sqrt{\left(\frac{\omega \mu_{0}}{2 \sigma_{i}}\right)} \quad i=x, y
$$

where $\omega=2 \pi f$ and $\mu_{0}=4 \pi \times 10^{-7} \mathrm{H} / \mathrm{m}$. It should be noted that (3) is valid if the thickness of composite is much greater than skin depth and with $x$ and $y$ are the anisotropic axes of the composite material. In multilayer CFC ground planes, if the skin depth is much lower than the thickness of the first layer, the proposed solution can also be used by considering only the surface impedance model of the first layer. We derive the solution for an anisotropic ground plane, and for the isotropic case: $Z_{x}=Z_{y}=Z$. Without loss of generality, the electric source current distribution can be as assumed to be polarized along $x$. Fig. 2 shows that we divide the solution into region I for the dielectric layer and region II for the air above it. Then the source-free Maxwell equations are solved to calculate the $z$-components of EM fields in each region.

By defining two dimensional Fourier transform as

$$
\tilde{\boldsymbol{E}}\left(k_{x}, k_{y}, z\right)=\int_{-\infty}^{+\infty} \int_{-\infty}^{+\infty} \boldsymbol{E}(x, y, z) e^{-j k_{x} x} e^{-j k_{y} y} d x d y,
$$

the solution in the spectral domain for the $z$-component of the EM field in each region can be obtained as

$$
\begin{gathered}
\tilde{E}_{z 2}=A e^{-j k_{2}(z-h)} \quad \operatorname{Im}\left(k_{2}\right)<0 \\
\tilde{H}_{z 2}=B e^{-j k_{2}(z-h)} \quad \operatorname{Im}\left(k_{2}\right)<0 \\
\tilde{E}_{z 1}=C \cos k_{1}(z-h)+D \sin k_{1}(z-h) \\
\tilde{H}_{z 1}=M \cos k_{1}(z-h)+N \sin k_{1}(z-h)
\end{gathered}
$$

where

$$
\begin{aligned}
& k_{2}^{2}=k_{0}^{2}-\beta^{2} \\
& k_{1}^{2}=k_{e}^{2}-\beta^{2} \\
& \beta^{2}=k_{x}^{2}+k_{y}^{2} .
\end{aligned}
$$

where $k_{0}=\omega \sqrt{\mu_{0} \varepsilon_{0}}$ and $k_{e}=\omega \sqrt{\mu_{0} \varepsilon_{0} \varepsilon_{r}}$, and $A, B, C, D, M$, and $N$ are unknown coefficients. The other components of fields are obtained from the Maxwell equations using

$$
\begin{aligned}
& \tilde{E}_{x}=\left(\frac{j k_{x}}{\beta^{2}}\right) \frac{\partial \tilde{E}_{z}}{\partial z}+\left(\frac{\omega \mu_{0} k_{y}}{\beta^{2}}\right) \tilde{H}_{z} \\
& \tilde{E}_{y}=\left(\frac{j k_{y}}{\beta^{2}}\right) \frac{\partial \tilde{E}_{z}}{\partial z}-\left(\frac{\omega \mu_{0} k_{x}}{\beta^{2}}\right) \tilde{H}_{z} \\
& \tilde{H}_{x}=\left(\frac{j k_{x}}{\beta^{2}}\right) \frac{\partial \tilde{H}_{z}}{\partial z}-\left(\frac{\omega \varepsilon k_{y}}{\beta^{2}}\right) \tilde{E}_{z} \\
& \tilde{H}_{y}=\left(\frac{j k_{y}}{\beta^{2}}\right) \frac{\partial \tilde{H}_{z}}{\partial z}+\left(\frac{\omega \varepsilon k_{x}}{\beta^{2}}\right) \tilde{E}_{z} .
\end{aligned}
$$

Then, the unknown coefficients are calculated by imposing the following boundary conditions

$$
\begin{array}{ll}
E_{x 1}=E_{x 2} & \text { at } \quad z=h \\
E_{y 1}=E_{y 2} & \text { at } \quad z=h \\
H_{x 1}=H_{x 2} & \text { at } \quad z=h \\
H_{y 1}-H_{y 2}=\tilde{J}_{x} & \text { at } \quad z=h \\
E_{x 1}=-Z_{x} H_{y 1} & \text { at } \quad z=0 \\
E_{y 1}=Z_{y} H_{x 1} & \text { at } \quad z=0 .
\end{array}
$$

After a considerable algebraic manipulation, the coefficients inside the slab are obtained (see Appendix I). Since we are interested in electric fields at the air-dielectric interface, $\tilde{E}_{x}$ and $\tilde{E}_{y}$ at $z=h$ are obtained from (12) and (13) as 

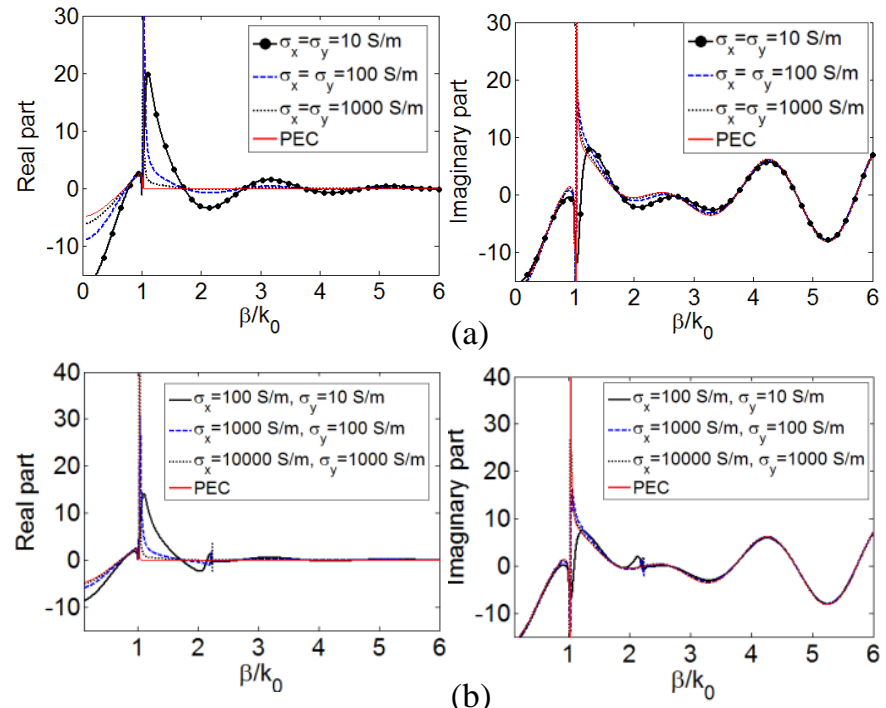

Fig. 3. The complex integrand $\tilde{F}_{r}$ for (a) isotropic and (b) anisotropic ground plane.

$$
\begin{aligned}
& \left.\tilde{E}_{x}\right|_{z=h}=\left(\frac{j k_{x} k_{1}}{\beta^{2}}\right) D+\left(\frac{\omega \mu_{0} k_{y}}{\beta^{2}}\right) N \\
& \left.\tilde{E}_{y}\right|_{z=h}=\left(\frac{j k_{y} k_{1}}{\beta^{2}}\right) D-\left(\frac{\omega \mu_{0} k_{x}}{\beta^{2}}\right) N .
\end{aligned}
$$

Note that by setting $Z_{x}=Z_{y}=0$ in (22) and (23), $\tilde{E}_{x}$ and $\tilde{E}_{y}$ become the coefficients for a PEC ground plane, as reported in the literature [16], [23]

$$
\begin{aligned}
& \left.\tilde{E}_{x}\right|_{z=h}=\left(\frac{-j}{\omega \varepsilon_{0}}\right)\left[\frac{-j k_{x}^{2} k_{1} k_{2}}{\beta^{2} T_{2}} \sin k_{1} h+\frac{k_{y}^{2} k_{0}^{2}}{\beta^{2} T_{3}} \sin k_{1} h\right] \tilde{J}_{x} \\
& \left.\tilde{E}_{y}\right|_{z=h}=\left(\frac{-j}{\omega \varepsilon_{0}}\right)\left[\frac{-j k_{y}^{2} k_{1} k_{2}}{\beta^{2} T_{2}} \sin k_{1} h+\frac{k_{x}^{2} k_{0}^{2}}{\beta^{2} T_{3}} \sin k_{1} h\right] \tilde{J}_{x} .
\end{aligned}
$$

By taking inverse Fourier transform from (22) and (23), the spatial domain electric field can be obtained as

$$
\begin{array}{r}
E_{i}(x, y)=\left(\frac{1}{4 \pi^{2}}\right) \int_{-\infty}^{+\infty} \int_{-\infty}^{+\infty} \tilde{E}_{i}\left(k_{x}, k_{y}\right) e^{j k_{x} x} e^{j k_{y} y} d k_{x} d k_{y} \\
i=x, y .
\end{array}
$$

\section{ELECTRIC FIELD CALCULATION}

Without loss of generality, only $E_{x}$ is considered for study. By applying the transformation of variables $k_{x}=\beta \cos \alpha$ and $k_{y}=\beta \sin \alpha,(26)$ is converted to

$$
E_{x}(x, y)=\left(\frac{1}{4 \pi^{2}}\right) \int_{0}^{+\infty} \int_{0}^{2 \pi} \tilde{E}_{x}(\beta, \alpha) e^{j x \beta \cos \alpha} e^{j y \beta \sin \alpha} \beta d \alpha d \beta
$$

so that by numerical integration with respect to $\alpha$, the electric field can be obtained from a single infinite integration as follows

$$
E_{x}(x, y)=\left(\frac{1}{4 \pi^{2}}\right) \int_{0}^{+\infty} \tilde{F}_{x}(\beta, x, y) d \beta
$$

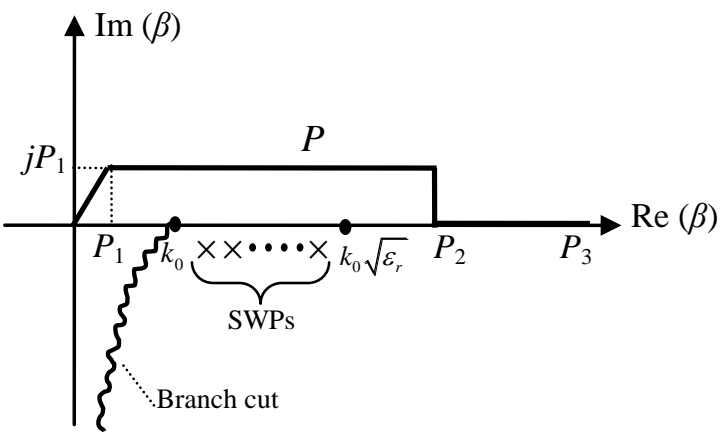

Fig. 4. The integration contour path in the complex $\beta$-plane.

where

$$
\tilde{F}_{x}(\beta, x, y)=\int_{0}^{2 \pi} \tilde{E}_{x}(\beta, \alpha) e^{j x \beta \cos \alpha} e^{j y \beta \sin \alpha} \beta d \alpha .
$$

The zeros of $\Delta$ (see (39), Appendix I) determine the poles of $\tilde{F}_{x}$, or the surface wave poles (SWP) of the structure. For the slab grounded by PEC, when the slab is electrically thin, $f<c_{0} / 4 h \sqrt{\varepsilon_{r}-1}$, there is only one SWP between $k_{0}$ and $k_{e}$ located at

$$
\beta=k_{0}\left[1+\left(\frac{1}{2}\right)\left(\frac{\varepsilon_{r}-1}{\varepsilon_{r}} k_{0} h\right)^{2}\right]
$$

which corresponds to the zero of $T_{2}$ [23]. For a substrate with small loss, the SWP has a small negative imaginary part. For a CFC ground plane that is highly conductive along the $y$ direction $\left(Z_{y} \rightarrow 0\right.$ ), it can be easily shown that the zeros of $T_{2}$ are also the zeros of $\Delta$. Hence, the same TM surface waves of the slab grounded by PEC are excited also in the slab with composite ground. Similarly, we may conclude that when HED is along $y$ direction and $Z_{x} \rightarrow 0$, the same SWPs exist when slab is grounded by CFC or PEC.

In order to calculate (28) for the slab with a CFC ground plane, first we study the behavior of integrand $\tilde{F}_{x}$ versus $\beta$ and compare it with the case that slab is grounded by PEC. The slab is chosen to be electrically thin and also with small loss, which is valid for many practical cases.

By choosing $f=5 \mathrm{GHz}, h=2.5 \mathrm{~mm}, \varepsilon_{r s}=5$, and $\tan \delta=$ $0.002, \quad \tilde{F}_{x}$ is evaluated at $(x, y)=(\lambda / 2,0)$ for different conductivity values for the ground plane as shown in Fig. 3. It is observed that by increasing the conductivity, the integrand gets closer to that of the slab grounded by PEC, showing an SWP at about $\beta=1.02 k_{0}$, which could be also calculated from (30). At the SWP location, $\tilde{F}_{x}$ shows a sharp change with noticeable magnitude. A small sharp change is also seen around $k_{e}$ for anisotropic composites. It is due to this fact that when $\beta \rightarrow k_{e}$, then $k_{1} \rightarrow 0$, setting the first term in $\Delta$ expression to zero which makes $\Delta$ to be a small non-zero value, specially when $\left(Z_{x}-Z_{y}\right)$ is small. However, $k_{1}=0$ is also a zero of the numerator of $D$ and $N$ coefficients. As a 

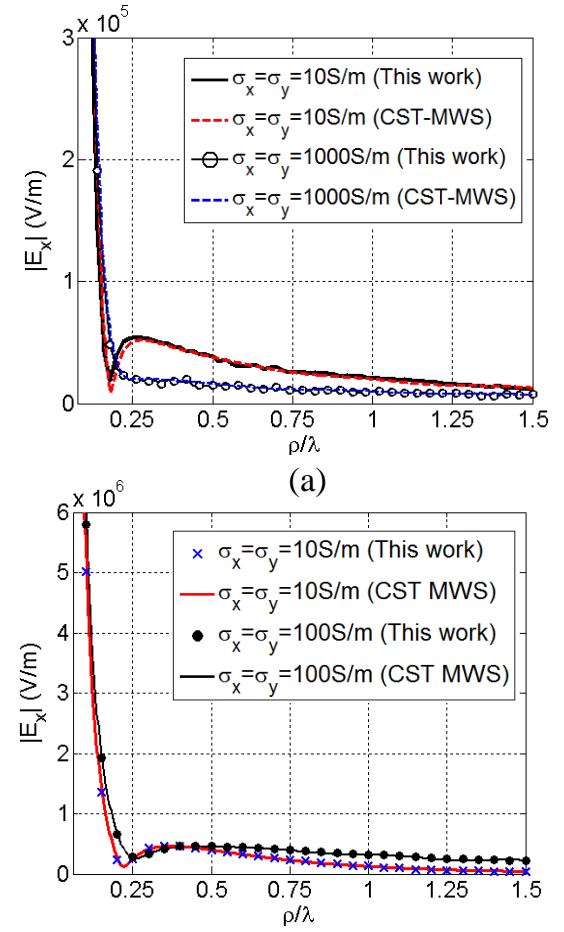

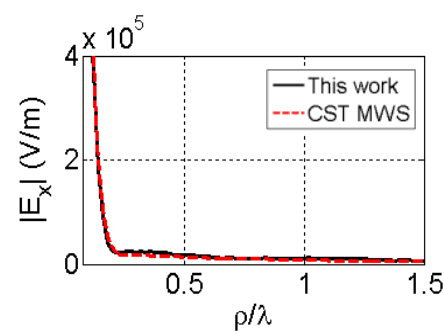

(c) (b)

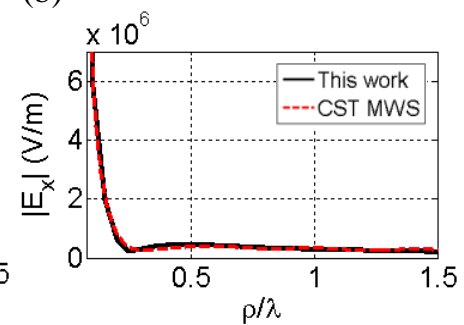

(d)
Fig. 5. The magnitude of $E_{x}$ along $x$-axis, (a) isotropic ground and $f=5 \mathrm{GHz}$, (b) isotropic ground and $f=10 \mathrm{GHz}$, (c) anisotropic ground with $\left(\sigma_{x}, \sigma_{y}\right)=(1000,100) \mathrm{S} / \mathrm{m}$ at $f=5 \mathrm{GHz},(\mathrm{d})$ anisotropic ground with $\left(\sigma_{x}\right.$, $\left.\sigma_{y}\right)=(1000,100)$ at $f=10 \mathrm{GHz}$.

result, a slightly sharp change of $\tilde{F}_{x}$ occurs around $\beta=k_{e}$.

In order to take the integration in (28) over the real axis of complex plane, some techniques like singularity extraction are needed to deal with SWPs [19], [20]. By increasing the frequency or the dielectric thickness, the number of SWPs may increase, leading to a complicated problem which includes locating the poles and performing the integration near them. Some works uses a deformed integration path to avoid dealing with SWPs [22]. Following [22], we consider the contour $P$ shown in Fig. 4 and defined by parameters $P_{1}, P_{2}$, and $P_{3}$. Without crossing the branch cut, the path fulfills the condition $\operatorname{Im}\left(k_{2}\right)<0$. There is no concern about $\operatorname{Im}\left(k_{1}\right)<0$ since (22) and (23) are even functions with respect to $k_{1}$. The path is deformed over $\left[k_{0}, k_{e}\right]$ so that no knowledge about SWP locations is required. Then it returns to the real axis at $P_{2}$ and is truncated at $P_{3}$ when convergence of the integral has been obtained.

Since the observation point and source are at the same level $(z=h)$, the integration in (28) converges slowly, which needs

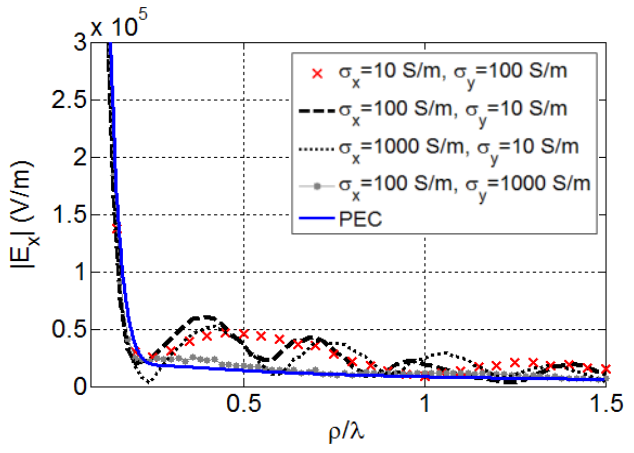

Fig. 6. The effect of ground anisotropy on $E_{x}$ at $f=5 \mathrm{GHz}$.

special treatment [16]-[20], [23]. The imaginary part of the integrand $\tilde{F}_{x}$ is oscillatory and diverges as $\beta$ increases, as shown in Fig. 3(b), making (28) converge slowly as beta increases. However, it should be noted that when $\beta \rightarrow \infty$ the behavior of both real and imaginary parts of $\tilde{F}_{x}$ for the slab with CFC ground plane converges to that of the slab grounded by PEC, independent of the CFC conductivity and anisotropy. The physical interpretation is that $\beta \rightarrow \infty$ implies $f \rightarrow 0$, hence from (3) we have $Z_{x}, Z_{y} \rightarrow 0$, making the ground plane to be like PEC. In the Appendix II, it is proven that the asymptotic form of $\tilde{E}_{x}$ for large $\beta$ is

$$
\left.\tilde{E}_{x}^{\infty}\right|_{C F C}=\left.\tilde{E}_{x}^{\infty}\right|_{P E C}=\left(\frac{-j}{2 \omega \varepsilon_{0}}\right)\left[\frac{k_{0}^{2} \varepsilon_{e f f}-k_{x}^{2}}{\varepsilon_{e f f} \beta}\right]
$$

where $\varepsilon_{\text {eff }}=\left(\varepsilon_{r}+1\right) / 2$ and superscript $\infty$ refers to $\beta \rightarrow \infty$. Therefore,

$$
\left.\tilde{F}_{x}^{\infty}\right|_{C F C}=\left.\tilde{F}_{x}^{\infty}\right|_{P E C}
$$

where $\tilde{F}_{x}^{\infty}$ is the asymptotic, or also called as the static part of $\tilde{F}_{x}$. We get advantage from the asymptotic equality in (32) to overcome the convergence problem of (28) as follows. The static part of the integrand in (28) is subtracted and added as

$$
\int_{0}^{+\infty} \tilde{F}_{x} d \beta=\int_{0}^{+\infty}\left[\tilde{F}_{x}-\left.\tilde{F}_{x}^{\infty}\right|_{C F C}\right] d \beta+\left.\int_{0}^{+\infty} \tilde{F}_{x}^{\infty}\right|_{C F C} d \beta
$$

so that in the right hand side of (33), the first term is a more rapidly converging integral, easily evaluated numerically. Substituting (32) in (33), the second term can be replaced by $I_{x}^{\infty}=\left.\int_{0}^{+\infty} \tilde{F}_{x}^{\infty}\right|_{P E C} d \beta$. Pozar showed that $I_{x}^{\infty}$ has a closedform expression as [24]

$$
I_{x}^{\infty}=\left(\frac{-j}{4 \pi \omega \varepsilon_{0} \varepsilon_{e f f}}\right)\left(\varepsilon_{e f f} k_{0}^{2}+\frac{\partial^{2}}{\partial x^{2}}\right)\left(\frac{e^{j k_{0} \sqrt{\varepsilon_{e f f}} R_{1}}}{R_{1}}-\frac{e^{j k_{0} \sqrt{\varepsilon_{e f f}} R_{2}}}{R_{2}}\right)
$$

where $R_{1}=\sqrt{x^{2}+y^{2}}$, and $R_{2}=\sqrt{x^{2}+y^{2}+4 h^{2}}$. In fact, (34) is the Green's function of an HED located at $(0,0, h)$ in the homogeneous medium with permittivity of $\varepsilon_{\text {eff }}$, and grounded by PEC at $z=0$. By replacing (34) in (33), the electric field can be calculated from (28). 
In order to calculate $E_{y}$, by replacing (23) in (26), the above procedure can be applied so that $I_{y}^{\infty}$ can be obtained from (34) by replacing $\varepsilon_{\text {eff }} k_{0}^{2}+\partial^{2} / \partial x^{2}$ with $\partial^{2} / \partial x \partial y$.

\section{NUMERICAL RESULTS}

In this section, we evaluate the electric field $E_{x}$ at the airdielectric interface for the ground plane with different conductivity characteristics, and at different frequencies. The strength of HED moment is one $(I d x=1)$. By using the slab parameters given in previous section, the magnitude of $E_{x}$ is calculated along $x$-axis at $y=0$ as shown in Fig. 5. The results are obtained at two operating frequencies, $f=5$ and $10 \mathrm{GHz}$. The results are compared with computations using CST MWS [25], which uses the finite integration technique (FIT) to solve the discretized Maxwell equations. Good agreement is seen. In the CST model, the $8 \lambda \times 8 \lambda$ slab and ground plane are terminated by PML layers to approximate the assumption of a slab of infinite extent. We used about 1,800,000 mesh cells in CST simulations to get accurate results.

Figure 6 shows the effect of conductivity tensor of the ground plane on $E_{x}$ at $z=h$. Some fluctuations is observed for low values of $\sigma_{x}$ and $\sigma_{y}$, but by increasing the conductivity in both directions, the electric field behavior moves toward that of the slab grounded by PEC. When $Z_{x}$ and $Z_{y}$ gets small, $Z_{x}-Z_{y}$ gets small, hence, the anisotropic characteristic of the ground plane vanishes, making the behavior of the structure like isotropic case.

\section{CONCLUSION}

The Green's function is calculated for an infinitesimal HED on a dielectric slab over a CFC ground plane having anisotropic conductivity. The Green's function is evaluated for carbon-composite materials: RCCF CFC which is highly anisotropic, and $\mathrm{CNT}$ CFC which has isotropic conductivity. Because these materials have high conductivity and small skin depth, they are efficiently modeled using the surface impedance. It is shown that the electromagnetic fields at the air-dielectric interface depend on both the conductivity and anisotropic characteristics of the composite ground plane. The Green's function presented here is versatile and can be used in the numerical modeling of microstrip structures with a CFC ground plane.

\section{APPENDIX I}

The coefficients $C, D, M$, and $N$ are obtained as

$$
\begin{aligned}
& C=\left(\frac{-\beta^{2} \tilde{J}_{x}}{\Delta}\right) \cdot\left[-\left(\omega^{2} \mu_{0}^{2} k_{x} k_{1} k_{2} \beta^{4} \cos k_{1} h\right) T_{3}\right. \\
& +\left(Z_{x} k_{x}^{2}+Z_{y} k_{y}^{2}\right)\left(-j \omega^{3} \mu_{0}^{2} \varepsilon_{0} \varepsilon_{r} k_{x} k_{2} \beta^{2} \sin k_{1} h\right) T_{3} \\
& -\left(Z_{x} k_{y}^{2}+Z_{y} k_{x}^{2}\right)\left(-j \omega \mu_{0} k_{x} k_{1}^{2} k_{2} \beta^{2} \cos k_{1} h\right) T_{4} \\
& -Z_{x} Z_{y}\left(\omega^{2} \mu_{0} \varepsilon_{0} \varepsilon_{r} k_{x} k_{1} k_{2} \beta^{4} \sin k_{1} h\right) T_{4} \\
& \left.+\left(Z_{x}-Z_{y}\right)\left(\omega^{3} \mu_{0}^{2} \varepsilon_{0} k_{x} k_{y}^{2} k_{1}^{2} \beta^{2}\right)\right] \\
& D=\left(\frac{\beta^{2} \tilde{J}_{x}}{\Delta}\right) \cdot\left[-\left(\omega^{2} \mu_{0}^{2} k_{x} k_{1} k_{2} \beta^{4} \sin k_{1} h\right) T_{3}\right. \\
& +\left(Z_{x} k_{x}^{2}+Z_{y} k_{y}^{2}\right)\left(j \omega^{3} \mu_{0}^{2} \varepsilon_{0} \varepsilon_{r} k_{x} k_{2} \beta^{2} \cos k_{1} h\right) T_{3} \\
& -\left(Z_{x} k_{y}^{2}+Z_{y} k_{x}^{2}\right)\left(j \omega \mu_{0} k_{x} k_{1}^{2} k_{2} \beta^{2} \sin k_{1} h\right) T_{4} \\
& +Z_{x} Z_{y}\left(\omega^{2} \mu_{0} \varepsilon_{0} \varepsilon_{r} k_{x} k_{1} k_{2} \beta^{4} \cos k_{1} h\right) T_{4} \\
& \left.+\left(Z_{x}-Z_{y}\right)\left(j \omega^{3} \mu_{0}^{2} \varepsilon_{0} \varepsilon_{r} k_{x} k_{y}^{2} k_{1} k_{2} \beta^{2}\right)\right] \\
& M=\left(\frac{-\beta^{2} \tilde{J}_{x}}{\Delta}\right) \cdot\left[-\left(\omega^{3} \mu_{0}^{2} \varepsilon_{0} k_{y} k_{1} \beta^{4} \cos k_{1} h\right) T_{2}\right. \\
& +\left(Z_{x} k_{x}^{2}+Z_{y} k_{y}^{2}\right)\left(j \omega^{4} \mu_{0}^{2} \varepsilon_{0}^{2} \varepsilon_{r} k_{y} \beta^{2} \cos k_{1} h\right) T_{1} \\
& -\left(Z_{x} k_{y}^{2}+Z_{y} k_{x}^{2}\right)\left(j \omega^{2} \mu_{0} \varepsilon_{0} k_{y} k_{1}^{2} \beta^{2} \sin k_{1} h\right) T_{2} \\
& -Z_{x} Z_{y}\left(\omega^{3} \mu_{0} \varepsilon_{0}^{2} \varepsilon_{r} k_{y} k_{1} \beta^{4} \sin k_{1} h\right) T_{1} \\
& \left.-\left(Z_{x}-Z_{y}\right)\left(j \omega^{2} \mu_{0} \varepsilon_{0} \varepsilon_{r} k_{1} k_{2}^{2} k_{x}^{2} k_{y} \beta^{2}\right)\right] \\
& N=\left(\frac{\beta^{2} \tilde{J}_{x}}{\Delta}\right) \cdot\left[\left(\omega^{3} \mu_{0}^{2} \varepsilon_{0} k_{y} k_{1} \beta^{4} \sin k_{1} h\right) T_{2}\right. \\
& +\left(Z_{x} k_{x}^{2}+Z_{y} k_{y}^{2}\right)\left(j \omega^{4} \mu_{0}^{2} \varepsilon_{0}^{2} \varepsilon_{r} k_{y} \beta^{2} \sin k_{1} h\right) T_{1} \\
& -\left(Z_{x} k_{y}^{2}+Z_{y} k_{x}^{2}\right)\left(j \omega^{2} \mu_{0} \varepsilon_{0} k_{y} k_{1}^{2} \beta^{2} \cos k_{1} h\right) T_{2} \\
& -Z_{x} Z_{y}\left(\omega^{3} \mu_{0} \varepsilon_{0}^{2} \varepsilon_{r} k_{y} k_{1} \beta^{4} \cos k_{1} h\right) T_{1} \\
& \left.-\left(Z_{x}-Z_{y}\right)\left(\omega^{2} \mu_{0} \varepsilon_{0} \varepsilon_{r} k_{1}^{2} k_{2} k_{x}^{2} k_{y} \beta^{2}\right)\right]
\end{aligned}
$$

where

$$
\begin{aligned}
\Delta=\left(\beta^{6} \omega^{2} \mu_{0} \varepsilon_{0}\right) \cdot[ & {\left[\left(j \omega \mu_{0} T_{3}+Z_{x} k_{1} T_{4}\right)\left(k_{1} T_{2}-Z_{y} j \omega \varepsilon_{0} \varepsilon_{r} T_{1}\right)\right.} \\
& \left.+k_{x}^{2} T_{2} T_{4}\left(Z_{x}-Z_{y}\right)\right] \\
T_{1}= & k_{1} \cos k_{1} h+j \varepsilon_{r} k_{2} \sin k_{1} h \\
T_{2}= & k_{1} \sin k_{1} h-j \varepsilon_{r} k_{2} \cos k_{1} h \\
T_{3}= & k_{1} \cos k_{1} h+j k_{2} \sin k_{1} h \\
T_{4}= & -k_{1} \sin k_{1} h+j k_{2} \cos k_{1} h .
\end{aligned}
$$

Not shown here, the coefficients $A$ and $B$ can be easily calculated from (16) and (17). The effect of ground plane anisotropy is represented by terms including $Z_{x}-Z_{y}$ in (35)(39).

\section{APPENDIX II}

When $\beta \rightarrow \infty$, we have $\quad k_{1} \rightarrow j \beta, \quad k_{2} \rightarrow-j \beta$, 
$\sin k_{1} h \rightarrow j e^{\beta h} / 2$, and $\cos k_{1} h \rightarrow e^{\beta h} / 2$. By calculating the asymptotic values of $D$ and $N$ from (36) and (38) as

$$
\begin{gathered}
D^{\infty}=\left(\frac{\beta^{2} \tilde{J}_{x} e^{2 h \beta}}{\left.2 \Delta\right|_{\beta \rightarrow \infty}}\right) \cdot\left[\left(\omega^{2} \mu_{0}^{2} k_{x} \beta^{7}\right)+Z_{x} Z_{y}\left(\omega^{2} \mu_{0} \varepsilon_{0} \varepsilon_{r} k_{x} \beta^{7}\right)\right. \\
+\left(Z_{x} k_{x}^{2}+Z_{y} k_{y}^{2}\right)\left(j \omega^{3} \mu_{0}^{2} \varepsilon_{0} \varepsilon_{r} k_{x} \beta^{4}\right) \\
\left.-\left(Z_{x} k_{y}^{2}+Z_{y} k_{x}^{2}\right)\left(j \omega \mu_{0} k_{x} \beta^{6}\right)\right]
\end{gathered}
$$

$$
\begin{gathered}
N^{\infty}=\left(\frac{\beta^{2} \tilde{J}_{x} e^{2 h \beta} \varepsilon_{e f f}}{\left.2 \Delta\right|_{\beta \rightarrow \infty}}\right) \cdot\left[\left(\omega^{3} \mu_{0}^{2} \varepsilon_{0} k_{y} \beta^{6}\right)\right. \\
+\left(Z_{x} k_{x}^{2}+Z_{y} k_{y}^{2}\right)\left(j \omega^{4} \mu_{0}^{2} \varepsilon_{0}^{2} \varepsilon_{r} k_{y} \beta^{3}\right) \\
-\left(Z_{x} k_{y}^{2}+Z_{y} k_{x}^{2}\right)\left(j \omega^{2} \mu_{0} \varepsilon_{0} k_{y} \beta^{5}\right) \\
\left.+Z_{x} Z_{y}\left(\omega^{3} \mu_{0} \varepsilon_{0}^{2} \varepsilon_{r} k_{y} \beta^{6}\right)\right]
\end{gathered}
$$

and after some mathematical manipulation, $\tilde{E}_{x}^{\infty}$ is obtained from (22) as

$$
\begin{gathered}
\tilde{E}_{x}^{\infty}=\tilde{J}_{x} \frac{\left(k_{0}^{2} \varepsilon_{\text {eff }} k_{y}^{2}-\beta^{2} k_{x}^{2}\right)\left(\omega \mu_{0} \beta^{3} e^{2 h \beta}\right)}{\left.2 \Delta\right|_{\beta \rightarrow \infty}} \times \\
{\left[-j \beta^{4} Z_{x}+\beta^{2} Z_{y}\left(j \omega^{2} \mu_{0} \varepsilon_{0} \varepsilon_{r}\right)\right.} \\
+Z_{x} Z_{y} \omega \varepsilon_{0} \varepsilon_{r} \beta^{3}+\omega \mu_{0} \beta^{3} \\
\left.\left(Z_{x}-Z_{y}\right) k_{x}^{2}\left(j \omega^{2} \mu_{0} \varepsilon_{0} \varepsilon_{r}+j \beta^{2}\right)\right]
\end{gathered}
$$

where $\left.\Delta\right|_{\beta \rightarrow \infty}$ is the asymptotic value of (39) given by

$$
\begin{aligned}
\left.\Delta\right|_{\beta \rightarrow \infty} & =\beta^{6} \omega^{2} \mu_{0} \varepsilon_{0}\left(\frac{\varepsilon_{\text {eff }}}{2}\right) e^{2 h \beta} \\
& \times\left[-\beta^{2}\left(\beta Z_{x}+j \omega \mu_{0}\right)\left(-j \omega \varepsilon_{0} \varepsilon_{r} Z_{y}-\beta\right)-\beta^{2} k_{x}^{2}\left(Z_{x}-Z_{y}\right)\right] .
\end{aligned}
$$

By considering the highest degree terms in the numerator and denominator of (46) and after more simplifying, $\tilde{E}_{x}^{\infty}$ is converted to

$$
\tilde{E}_{x}^{\infty}=\tilde{J}_{x} \frac{\left(k_{0}^{2} \varepsilon_{e f f}-k_{x}^{2}\right)\left(-j Z_{x} \beta^{2}\right)}{2 \omega \varepsilon_{0} \varepsilon_{e f f} Z_{x} \beta^{3}}=\tilde{J}_{x}\left(\frac{-j}{2 \omega \varepsilon_{0}}\right) \frac{\left(k_{0}^{2} \varepsilon_{e f f}-k_{x}^{2}\right)}{\varepsilon_{e f f} \beta} .
$$

When the slab is grounded by PEC, $\tilde{E}_{x}^{\infty}$ is obtained directly from (24), which can be easily shown that it is equal to (48).

\section{ACKNOWLEDGMENT}

The authors would like to thank Dr. I. D. Rosca and Prof. S. V. Hoa from Concordia Center for Composites (CONCOM) for providing the carbon fiber composite samples, and also for useful discussions about composite materials.

\section{REFERENCES}

[1] M. H. Choi, B. H. Jeon, and I. J. Chung, "The effect of coupling agent on electrical and mechanical properties of carbon fiber/phenolic resin composites," Polymer, vol. 41, no. 9, pp. 3243-3252, 2000.
[2] A. L. Bogorad, M. P. Deeter, K. A. August, G. Doorley, J. J. Likar, and R. Herschitz," Shielding effectiveness and closeout methods for composite spacecraft structural panels," IEEE Trans. Electromagn. Compat., vol. 50, no. 3, pp. 547-555, 2008.

[3] C. L. Holloway, M. S. Sarto, and M. Johansson, "Analyzing carbonfiber composite materials with equivalent-layer models," IEEE Trans. Electromagn. Compat., vol. 47, no. 4, pp. 833-844, 2005.

[4] I. M. De Rosa, F. Sarasini, M. S. Sarto, and A. Tamburrano, "EMC impact of advanced carbon fiber/carbon nanotube reinforced composites for next-generation aerospace applications," IEEE Trans. Electromagn. Compat., vol. 50, no. 3, pp. 556-563, 2008.

[5] Y. Huang, N. Li, Y. Ma, F. Du, F. Li, X. He, X. Lin, H. Gao, and Y. Chen, "The influence of single-walled carbon nanotube structure on the electromagnetic interference shielding efficiency of its epoxy composites," Carbon, vol. 45, no. 8, pp.1614-1621, 2007.

[6] J. B. Bai, and A. Allaoui, "Effect of the length and the aggregate size of MWNTs on the improvement efficiency of the mechanical and electrical properties of nanocomposites - experimental investigation," Composites Part A, vol. 34, no. 8. pp. 689-694, 2003.

[7] I. D. Rosca, and S. V. Hoa, "Highly conductive multiwall carbon nanotube and epoxy composites produced by three-roll milling," Carbon, vol. 47, no. 8, pp. 1958-1968, 2009.

[8] P. J. Burke, "An RF circuit model for carbon nanotubes," IEEE Trans. Nanotechnol., vol. 2, no. 1, pp. 55-58, 2003.

[9] A. Mehdipour, A.-R. Sebak and C. W. Trueman, and S. V. Hoa "Carbon-Fiber composite T-match folded bow-tie antenna for RFID applications," presented at the IEEE Antenna and Propagation Symp. (APS 2009), Charleston, SC, Jun. 1-5, 2009.

[10] A. Mehdipour, A.-R. Sebak, C. W. Trueman, I. D. Rosca, and S. V. Hoa, "Reinforced continuous carbon-fiber composites using multi-wall carbon nanotubes for wideband antenna applications," IEEE Trans. on Antenna and Propagat., vol. 58, no. 7, pp. 2451-2456, 2010.

[11] P. J. Burke, and C. Rutherglen, "Carbon nanotube based variable frequency patch antenna", US patent 2009/0231205 A1, September 17, 2009.

[12] A. Mehdipour, I. D. Rosca, A.-R. Sebak, C. W. Trueman, and S. V. Hoa, "Full-composite fractal antenna using carbon nanotubes for multiband wireless applications," IEEE Antenna and Wireless Propagation Lett., vol. 9, pp. 891-894, 2010.

[13] W. Hoenlein, F. Kreupel, G. S. Duesberg, A. P. Graham, M. Liebau, R. V. Seidel, and E.Unger, "Carbon nanotube applications in microelectronics," IEEE Trans. Compon. Packag. Techn., vol. 27, no. 4, pp. 629-634, 2004.

[14] L. Josefsson, and P. Persson, Conformal Array Antenna Theory and Design. Piscataway, NJ: IEEE Press, 2006.

[15] J.-F. Kiang, "Rectangular patch resonator with laminated ground plane," IEEE Trans. on Antenna and Propagat., vol. 43, no. 12, pp. 1361-1368, 1995.

[16] V. S. Reddy and R. Garg, "Efficient analytical evaluation of the asymptotic part of Summerfeld type reaction integrals in microstrip/slot structures," IEE Microw. Antenna. Propag. vol. 147, no. 1, 2000.

[17] G.-W. Pan, J. Tan, and J. D. Murphy, "Full-wave analysis of microstrip floating-line discontinuities," IEEE Trans. Electromagn. Compat., vol. 36, no. 1, pp. 49-58, 1994.

[18] I. E. Rana, and N. Alexopoulos, "Current distribution and input impedance of printed dipoles," IEEE Trans. on Antenna and Propagat., vol. 29, no. 1, pp. 99-105, 1981.

[19] D. Pozar, "Input impedance and mutual coupling of rectangular microstrip antennas," IEEE Trans. Antennas Propagat., vol. 30, no. 6, pp. 1191-1196, 1982.

[20] J. R. Mosig, T. K. Sarkar, "Comparison of quasi-static and exact electromagnetic fields from a horizontal electric dipole above a lossy dielectric backed by an imperfect ground plane" IEEE Trans. Microwave Theory Tech., vol. 34, no. 4, pp. 379-387, 1986.

[21] "Concordia Center for Composites (CONCOM)," Concordia University, QC, Canada, 1979 [Online]. Available: http://concom.encs.concordia.ca.

[22] E. H. Newman, and D. Forrai, "Scattering from a microstrip patch" IEEE Trans. Antennas Propagat., vol. 35, no. 3, pp. 245-251, 1987.

[23] T. Itoh, Ed., Numerical Techniques for Microwave and MillimeterWave Passive Structure. New York: Wiley, 1989, ch. 9. 
[24] D. Pozar, "Improved computational efficiency for the moment method solution of printed dipoles and patches," Electromagnetics, vol. 3, pp. 299-309, 1983.

[25] CST - Microwave Studio, Computer Simulation Technology, 2009.

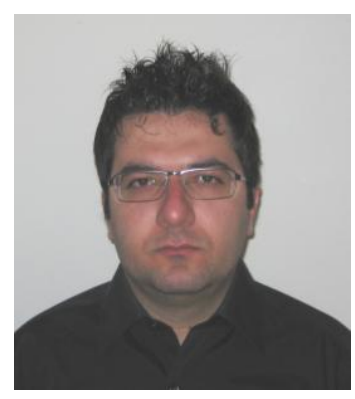

Aidin Mehdipour received the B.S. degree from Amirkabir University of Technology, Tehran, Iran, in 2003, the M.S. degree from the University of Tehran, Tehran, Iran, in 2006, and the Ph.D. degree from Concordia University, Montreal, QC, Canada, in 2011, all in electrical engineering. He is currently a Postdoctoral Fellow with the Institute National de la Recherche Scientifique (INRS), Montreal, QC, Canada. His main research interests include advanced carbon fiber composites, novel material development for shielding and antenna/microwave applications, EMI/EMC, small antenna design, RFID, multiband, ultrawideband, and millimeter wave antennas.

Dr. Mehdipour has won several prestigious awards such as the Governor General's Gold Medal-Technology, Industry and the Environment, awarded by the government of Canada, and David J. Azrieli Graduate Fellowship, as the highest ranking fellowship award in Concordia University. $\mathrm{He}$ was awarded the France and André Desmarais Graduate Fellowship in 2010, the Howard Webster Foundation Doctoral Fellowship and Doctoral Thesis Completion Award in 2011. He is a member of IEEE AP and EMC societies and of the Applied Computational Electromagnetics Society (ACES).

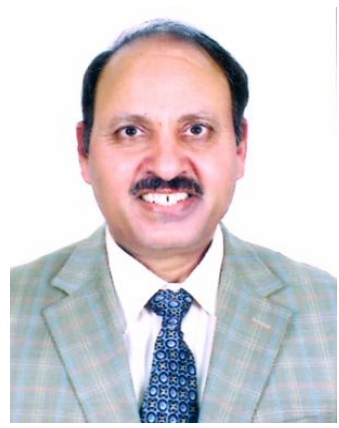

Abdel R. Sebak received the B.Sc. degree from Cairo University, the M.Eng. and Ph.D. degrees from the University of Manitoba all in electrical engineering.

From 1984 to 1986 , he was with the Canadian Marconi Company working on the design of microstrip phased array antennas. From 1987 to 2002, he was a Professor in the ECE Department, University of Manitoba. $\mathrm{He}$ is a Professor of Electrical and Computer Engineering, Concordia University, Montreal. His current research interests include phased array antennas, mm-wave imaging, computational electromagnetics, and interaction of EM waves with new materials and bio-electromagnetics.

Dr. Sebak received the 2000 and 1992 University of Manitoba Merit Award for outstanding Teaching and Research, the 1994 Rh Award for Outstanding Contributions to Scholarship and Research, and the 1996 Faculty of Engineering Superior Academic Performance. Dr. Sebak has served as Chair for the IEEE Canada Awards and Recognition Committee (2002-2004) and the Technical Program Chair ANTEM-2006.

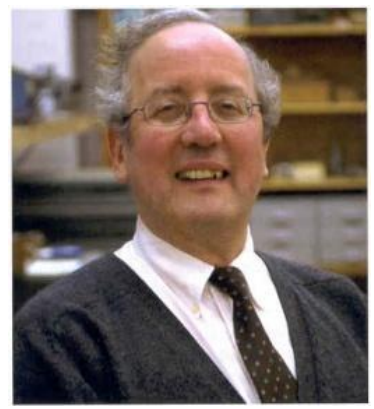

Christopher W. Trueman received his Ph.D. from McGill University in 1979. He has applied the methods of computational electromagnetics to problems such as aircraft antenna performance, antenna-to-antenna coupling and EMC on aircraft, aircraft and ship radar cross-section at $\mathrm{HF}$ frequencies, suppression of scattering of the signal of a commercial radio station from power lines, dielectric resonators, unconditionally-stable formulations for the finite-difference time-domain method, and the fields of portable radios such as cellular phones held against the head. Recently, his research has investigated carbon composite materials for aircraft and for building antennas, indoor propagation, and EMC issues between portable radios and medical equipment. Dr. Trueman is currently the Associate Dean for Academic Affairs in the Faculty of Engineering and Computer Science at Concordia University. 